Théologiques

Théologiques

\title{
La théologie face aux changements des représentations du cosmos : Quelle odyssée et pour quitter quels héritages?
}

\section{Pierre Gisel}

Volume 9, numéro 1, printemps 2001

Les cosmologies

URI : https://id.erudit.org/iderudit/005686ar

DOI : https://doi.org/10.7202/005686ar

Aller au sommaire du numéro

Éditeur(s)

Faculté de théologie de l'Université de Montréal

ISSN

1188-7109 (imprimé)

1492-1413 (numérique)

Découvrir la revue

Citer cet article

Gisel, P. (2001). La théologie face aux changements des représentations du cosmos : Quelle odyssée et pour quitter quels héritages ? Théologiques, 9(1), 17-48. https://doi.org/10.7202/005686ar
Résumé de l'article

La présente contribution cherche à saisir l'impact des changements de représentations du cosmos sur la théologie. Après avoir rappelé certaines données scientifiques et présenter un aperçu des données bibliques et chrétiennes en la matière, l'auteur fait une proposition de balisage théologique au regard de la non-homogénéité ou des contradictions qui se dégagent de la confrontation de la science et de la Bible. Il fait alors un retour sur les types d'articulation possibles entre le savoir scientifique et le fait de croire. Il présente enfin un aperçu touchant la question du statut de la théologie. 


\section{La théologie face aux changements des représentations du cosmos Quelle odyssée et pour quitter quels héritages?}

Pierre GISEL

Faculté de théologie Université de Lausanne

Nos connaissances sur le cosmos - ses origines, les lois de son déploiement, sa fin - changent. Nos manières d'éprouver le rapport de l'humain au monde en sont affectées, comme les représentations que nous pouvons en avoir aux plans psychologique, philosophique, culturel (dans les arts, la littérature, le cinéma, diverses œuvres de fiction, etc.)

Que l'on pense à la révolution psychologique et culturelle qu'a constitué le passage d'une représentation qui plaçait la terre au centre du cosmos et l'humain au centre de la terre, et qui, en outre, insérait l'ensemble dans une courte durée (au plus sept mille ans et non quinze milliards d'années par exemple), à un monde infini, disséminé ou pluriel, et peut-être en explosion. Plus près de nous, la théorie de la relativité ou la physique quantique, comme les travaux de ces dernières décennies sur le cerveau, ont aussi bousculé nos représentations du cosmos et des rapports de l'humain au monde et à la nature. En revisitant le passé de notre aire culturelle, l'Occident, du Proche-Orient ancien, de l'Égypte et de la Mésopotamie, puis de l'Antiquité grécoromaine, de l'Antiquité tardive et de l'aventure du christianisme, on s'aperçoit que l'histoire a traversé bien des changements de visions du monde et de paradigmes, elle a connu bien des acculturations ou des métissages socio-culturels. Ce n'est ni la première, ni la dernière fois que l'homme connaît de tels changements.

Foncièrement, sauf le fondamentalisme - un produit moderne au demeurant, inséparable d'une conjoncture marquée par les rationalismes des $\mathrm{XIX}^{\mathrm{e}}$ et $\mathrm{XX}^{\mathrm{e}}$ siècles - et l'aberration scientifique et religieuse 
qu'est le créationnisme ${ }^{1}$, la théologie chrétienne a toujours entrepris de remettre sur le métier sa compréhension de l'humain et du monde (il y a donc " odyssée »), et dès lors de repenser son propre discours, son statut, les limites de sa validité et sa portée. Non sans oscillations entre mouvements de réforme et nostalgies, innovations et relectures diverses du passé, protestations, tensions avec le présent et utopies. C'est qu'il n'y a pas de christianisme hors des jeux différenciés d'institutionnalisation et de contestation, et ce, dès les premiers siècles, au cœur du Moyen Âge, comme au long des Temps modernes, de la Renaissance aux Lumières et à la postmodernité.

Dans la présente contribution - délibérément synthétique vu l'ampleur des champs et des problèmes, et du coup de la bibliographie -, je me propose le parcours suivant : 1) un rappel des données scientifiques élémentaires, 2) un aperçu tout aussi élémentaire des données bibliques et chrétiennes à ce propos 3) une proposition de balisage théologique au regard de la non-homogénéité ou des contradictions qui se dégagent de la confrontation des deux premiers points, 4) un retour sur les types d'articulation possibles entre le savoir scientifique et le fait du croire, 5) un aperçu touchant la question du statut de la théologie.

\section{Rappel de données scientifiques}

L'univers a une histoire, celle de la matière qui s'organise. A partir d'un big bang de départ, selon la vision qui semble le mieux correspondre à l'état de nos connaissances scientifiques et à l'ensemble des observations faites jusqu'à présent, et au gré d'une évolution qui voit l'univers se refroidir, se dilater et se structurer, à très petite échelle (le monde des particules) comme à très grande échelle (les galaxies et les amas de galaxies). C'est progressivement que se prépare pour ainsi dire un berceau dans lequel la vie va éclore, à quoi il faut ajouter l'idée qui s'impose aujourd'hui d'une continuité entre l'évolution de l'univers et celle de la vie, donc entre l'inerte et le vivant.

1. Pour ma position sur ce sujet, je renvoie au livre écrit avec Lucie KAENNEL, La création du monde, Genève-Bienne, Labor et Fides-Société biblique suisse, 1999, p. 111-118, livre qui se tient par ailleurs en l'arrièreplan de certaines sections du présent article. 
On sait par ailleurs que les théories fixistes (pour exemple, Georges Cuvier, 1769-1832) ont fait place à des transformismes. Le nom de Charles Darwin (1809-1882) est ici emblématique, avec la théorie évolutionniste. On considère aujourd'hui comme faits biologiques acquis qu'il y a croissance exponentielle du vivant, que seule une petite partie des individus survit et se reproduit, que la destruction se fait selon un processus sélectif (sélection naturelle). Enfin, il importe de souligner que pas plus qu'il ne peut être question d'établir un commencement de la vie, il n'est véritablement possible de déterminer un commencement de l'homme, l'évolution étant en outre de toute manière non linéaire.

Pour résumer, on peut dire que la complexification, la structuration, l'adaptabilité, l'irréversibilité, le facteur temps, les phénomènes de sélection sont autant de paramètres qui entrent en ligne de compte dans une évolution de la vie fortement marquée par l'environnement.

Touchant l'humain, il convient d'ajouter des considérations relatives au fonctionnement du cerveau. En effet, on a affaire ici à ce qui représente le point extrême de l'évolution dont le cerveau humain garde secrètement la mémoire accumulée. De plus, on touche là certaines des avancées les plus neuves de la recherche scientifique contemporaine, avancées qui posent des questions redoutables à la foi, à la théologie, et plus largement, à la culture et aux sociétés.

Les travaux récents en biologie moléculaire et à propos des mécanismes du cerveau ont enregistré des résultats spectaculaires ${ }^{2}$. Ils cassent en tout cas tout reste de dualisme corps/esprit, tout dualisme entre les cellules du cerveau et le cerveau comme siège d'un esprit ou d'un sujet libre.

À propos du cerveau, on peut distinguer un plan anatomique : qui se rapporte aux connexions et réseaux neuronaux (ordre de grandeur : cent milliards de neurones); un plan physiologique qui concerne l'activité électrique et les signaux chimiques circulant dans le

2. Cf., par exemple, Jean-Pierre Changeux, L'homme neuronal, Paris, Fayard, 1991 (1983). Pour un débat touchant les questions que cela pose quant à la compréhension qu'un humain peut avoir de lui-même : Jean-Pierre ChangeuX et Paul Ricenur, Ce qui nous fait penser. La nature et la règle, Paris, Odile Jacob, 1998. 
cerveau ; un plan comportemental qui permet de soutenir que le cerveau fonctionne de façon projective, faisant des hypothèses sur le monde et les mettant à l'épreuve. Notons que l'ensemble du fonctionnement fait l'objet d'expériences minutieuses. On peut mentionner, d'abord, les lésions partielles et l'observation systématique et différenciée des dysfonctionnements qui y sont liés ; ils touchent la parole, la perception sensorielle, la planification des actions, la perturbation de l'image de soi, le rapport à autrui. Signalons aussi l'imagerie cérébrale, qui permet d'établir expérimentalement une distribution différentielle des activités électriques et chimiques des territoires cérébraux, en lien avec de la souffrance (réelle ou imaginée) ou des états dépressifs, schizophréniques ou hallucinatoires. On ajoutera les expérimentations électrophysiologiques en rapport avec l'activité des neurones, faisant voir des liens entre une activité neuronale et ce qui est vécu subjectivement.

Tout cela conduit à une inscription assez radicale des faits psychiques dans l'ordre de la nature ou de la matière. $\mathrm{Si}$, comme le rappelle le philosophe Paul Ricœur, on ne peut pas dire, en rigueur, que le cerveau pense (penser suppose la conscience, réflexive, d'un sujet), on doit au moins admettre que lorsque je pense, il se passe quelque chose dans mon cerveau.

Au total, le cerveau est complexe : fait d'entités distinctes, en rapport non selon continuité de circulation, mais par communications discontinues (des synapses : cent mille contacts discontinus par neurone, un demi-milliard par centimètre cube, soit un nombre de combinaisons quasi-infinies). Il est hiérarchisé : il enregistre et analyse l'information par plusieurs voies parallèles, d'où une activité de synthèse. Il développe enfin une activité spontanée : il est projectif, avec ce que cela suppose de construction et d'organisation. On peut aussi observer que ce qui concerne le rapport à l'autre, rapport d'empathie ou de sympathie, se joue au niveau du cortex frontal, la partie évolutivement la plus récente.

Dans l'histoire de la vie, les modifications internes au cerveau prennent le relais de l'évolution des espèces. S'en trouve constitué un appareil en interaction avec un environnement non seulement physique, mais aussi social et culturel. Concrètement, le petit d'homme, dont le cerveau est encore incomplètement stable, passe par un apprentissage. Une mémoire est ici à l'œuvre, une mémoire primaire ou immédiate, 
mémoire de travail présente, et une mémoire secondaire, inconsciente, qui apparaît maintenue en ligne pendant le travail de la première ; et qui est lourde, notamment, de composantes émotionnelles.

Dans nos gènes et nos cellules sont largement inscrits des héritages qui remontent aux origines de la vie. Avec l'homme, une évolution génétique laissée en suspens apparaît prolongée sur un mode non génétique (social et culturel) : le socialement approprié trouve son inscription dans le cerveau. L'auto-organisation du vivant, ici via le cerveau, continue à se faire dans un jeu avec le milieu, sur une base matérielle ayant intégré des mémoires de longue durée.

Partir en quête de nos commencements, c'est se retrouver face à un horizon on ne peut plus grand, avec tout ce que cela représente d'énigme : un quasi-infini s'ouvre à nous, qui nous dépasse. Mais, comme en écho, cet infiniment grand renvoie à l'infiniment petit sans lequel rien ne serait : l'univers s'est mis en branle parce que des particules élémentaires se sont assemblées pour passer par différents stades, selon un processus qui se poursuit de façon exponentielle, créant de nouvelles structures de plus en plus complexes, accueillant la vie et lui permettant de se déployer.

Mutatis mutandis, on pourrait ici faire écho à Pascal qui, dans les Pensées ${ }^{3}$, s'émerveille devant l'infiniment grand et l'infiniment petit entre lesquels prend place l'humain. Un infiniment grand et un infiniment petit qui, par ailleurs, semblent pour ainsi dire renvoyer à un fait de différence inscrit au cœur de l'évolution, comme donné en creux : l'histoire de l'univers est faite de différenciations, de complexifications, de spécifications, et la vie suppose la différence.

Il convient de souligner encore l'irréversibilité de l'évolution, à l'encontre de toute illusion de retour à un état initial : telle une spirale, l'évolution se déploie et se construit dans le temps et dans l'espace, montrant combien la vie - et donc aussi celle de l'homme - est insérée dans le processus d'un développement qui aurait pu être autre mais qu'on ne peut plus déconstruire. Pour anticiper, on peut dire que l'humain est inscrit en contingence - des données de fait -, il prend place dans un monde dont il hérite et qui le dépasse.

3. Pensée 72 selon la numérotation de l'édition de Brunschvicg, 199 selon celle de Lafuma. 
Remarquons enfin que si le discours scientifique s'inscrit dans l'ordre du vérifiable, du démontrable, de ce qui relève d'hypothèses et de lois mathématiques que les découvertes viennent confirmer ou mettre en cause, il n'en demeure pas moins que, sur certains points, ce discours relève d'extrapolations : les preuves et les vérifications ne sont pas toujours possibles. Surtout, chaque représentation ne peut se construire et chaque loi énoncée ne peut avoir validité qu'à partir et en fonction d'un commencement de l'univers déjà donné. L'astrophysicien Marc Lachièze-Rey le souligne : "Le processus fondateur de l'Univers, s'il en existe un, n'a pu se dérouler dans le cadre de l'Univers puisqu'il a abouti, précisément, à créer ce cadre. [...] la physique ne peut concevoir ce qui aurait pu se dérouler avant, que cet avant soit chronologique [...] ou fondateur, explicatif [...]. Un "autre univers" - par définition - ne pourrait avoir aucune interaction avec le nôtre. Sinon, il ferait partie du nôtre ${ }^{4}$. La science dit beaucoup de choses sur l'univers; elle ne peut en dire le dehors, pour autant, justement, que cela ait un sens.

\section{Aperçu de données bibliques et chrétiennes}

\subsection{Des cosmologies changeantes}

Chacun sait que la Bible présente, en son début, deux récits de création. La description des commencements et les représentations cosmologiques qu'ils suggèrent diffèrent. Ces deux textes ne viennent d'ailleurs pas de la même époque, et le second qui s'offre à la lecture, dans l'ordre biblique reçu, va chronologiquement avant celui qui le précède dans le texte final.

Dans le même ensemble biblique, on peut lire des textes qui offrent d'autres images encore du commencement du monde et de ce qui lui assure stabilité. Ainsi, en est-il du Ps 104,5 avec la représentation de fondements, voire de colonnes, sur lesquels reposerait la terre (Dieu a " établi la terre sur ses fondements ») ou, de Job 38, 4-6, Dieu interroge : "Où étais-tu quand je fondais la terre ? [...] Qui en a fixé les mesures, le sais-tu ? [...] Dans quoi ses bases sont-elles enfoncées ? »), ou encore la mention d'un "sein maternel » (Job 38, 8 et 16: "Qui a fermé la mer avec des portes, quand elle s'élança et sortit du sein

4. Marc LACHIÈZE-REY, "Les origines ", dans Recherches de science religieuse 81 (1993), p. 539-557, ici : p. 546s. 
maternel ? [...] Es-tu parvenu jusqu'aux sources de la mer ? T'es-tu promené dans les profondeurs de l'abîme ? »).

En termes de représentations toujours, on a pu parler de combat originel et créateur entre Dieu et des forces hostiles ou au contraire d'un acte décisivement souverain de Dieu, par delà toute mesure humaine ou terrestre, avec, parfois, des figures médiatrices proches de Dieu.

Pour le premier registre, on lira notamment les Psaumes, 74, 1214 : «Dieu, tu es mon roi dès l'origine, auteur des victoires au milieu de la terre. C'est toi qui as cassé par ta force la mer, tu as brisé les têtes du dragon sur les eaux. C'est toi qui as fracassé les têtes de Léviathan, tu l'as donné à manger au peuple des bêtes sauvages »; ou 89, 11 : "C'est toi qui as écrasé Rahab, comme un cadavre percé de coups " (selon d'autres représentations, c'est Dieu qui a formé le Léviathan «pour jouer avec lui », 104, 26 ; cf. aussi Job 40, 15 et 25).

Pour le second registre, on peut renvoyer à Ésaïe 40, 12-18 : «Qui a mesuré les eaux dans le creux de sa main, fixé les dimensions des cieux avec la paume, celle de toute la poussière de la terre dans un tiers de mesure ? [...] Qui a fixé une mesure à l'esprit de l'Éternel [...] ? [...] À qui voulez-vous comparer Dieu ?" (cf. aussi Job 11, 7-9) ; 44, 24 : "Ainsi parle l'Éternel qui te rachète, celui qui t'a façonné dès ta naissance : “Moi, l'Éternel, je fais toutes choses, seul je déploie les cieux, de moi-même j'étends la terre" »; enfin 45, 7: "Je forme la lumière et je crée les ténèbres ", voire le "malheur" (cf. 54, 16).

Quant aux figures médiatrices proches de Dieu, accompagnant plutôt le second registre - comme si se conjugaient une création cosmique hors mesure humaine et un principe secret qui la recueille de l'intérieur pour en faire une création pour la vie - , on mentionnera tout spécialement la Sagesse, " artisane » de la création (Sagesse 7, 22 et 8,6$)$ et proche de Dieu $(9,9$ : "Près de toi se tient la Sagesse qui connaît tes œuvres, et qui était présente lorsque tu créais le monde »). La même Sagesse précédant la création et présidant à la création se trouve en Proverbes 8, 22-31 : "J'ai été établie depuis l'éternité, dès le commencement, avant l'origine de la terre. J'ai été enfantée quand il n'y avait point d'abîmes [...]. Lorsqu'Il disposa les cieux, j'étais là ; [...] j'étais à l'œuvre auprès de lui, et je faisais de jour en jour ses délices, jouant devant lui tout le temps ", ou en Siracide 24, 1-22 :

Je suis sortie de la bouche du Très-Haut et comme une vapeur j'ai recouvert la terre. J'habitais dans les hauteurs du ciel et mon trône reposait 
sur la colonne de nuée. Le cercle du ciel, je l'ai parcouru, moi seule, et j'ai marché dans la profondeur des abîmes. [...] Avant que le temps ne commence, il m'a créée, et pour les siècles je ne cesserai pas d'exister. [...] Venez à moi, vous qui me désirez, et rassasiez-vous de mes fruits.

Un motif analogue et une structure semblable se retrouvent dans le Nouveau Testament, à propos du Christ cette fois, en Colossiens 1, 15ss. : "Il est l'image du Dieu invisible, le premier-né de toute la création. Car en lui tout a été créé dans les cieux et sur la terre, ce qui est visible et ce qui est invisible, trônes, souverainetés, principautés, pouvoirs. Tout a été créé par lui et pour lui. Il est avant toutes choses, et tout subsiste en lui », ou Éphésiens 1, 4-10 : "En lui, Dieu nous a élus avant la fondation du monde [...]. Il nous a fait connaître le mystère de sa volonté [...] : réunir sous un seul chef, le Christ, tout ce qui est dans les cieux et ce qui est sur la terre ", ou encore Hébreux 1,2 : «C'est par lui [le Fils] qu'il a fait les mondes » et Jean 1, 1-4 : «Au commencement était la Parole [...]. Tout a été fait par elle, et rien de ce qui a été fait n'a été fait sans elle ». Rappelons que la littérature rabbinique soutient que Dieu regarda la Tora pour savoir comment créer le monde : même dispositif, qui voit le moment central et structurant de l'histoire être placé au cœur même de l'origine du monde.

Quant à la réception de la Bible que sanctionnent les théologies chrétiennes, elle suppose elle aussi, au cours du temps, des représentations changeantes, que l'arrière-fond soit plus stoïcien (avec la consécration chrétienne d'une loi naturelle), néo-platonicien (avec ses reprises dans l'anagogie ou la mystique chrétienne), aristotélicien (avec les différenciations internes de l'être qui l'accompagnent, ainsi que des topologies et divers ordonnancements de qualités essentielles) ou, à l'aube des Temps modernes, celui de lois mathématisables et de systèmes mécaniques (pouvant générer diverses théodicées), celui d'une nature sans mystère intrinsèque (lié à un Dieu cause directe ou, au contraire, inconnu et autre), voire, plus récemment, celui d'une nature faite d'énergies et d'un devenir dont Dieu pourrait être partie prenante, ou tout au moins partie impliquée.

On le pressent, si la théologie chrétienne se comprend à la fois comme tradition - et non suite discontinue de représentations diverses - et entend se référer à des Écritures antérieures qu'elle reçoit comme fondement, malgré leur diversité interne, il faut qu'elle ait opéré quelque déplacement. Elle aura notamment dissocié la question 
de la vérité de celle des représentations du monde, de l'humain et de Dieu au travers desquelles s'exprime cette vérité : la vérité est dite à travers des représentations (il n'y a pas en ces matières de vérité pensable hors représentations supposées, même si les deux ordres sont distincts), mais sans faire nombre avec elles (la vérité ne s'y résorbe pas, ni ne s'y épuise pas). Traversant et assumant une diversité de représentations cosmologiques, renvoyant à une Bible qui a ellemême consacré une telle diversité, la théologie chrétienne ne peut que révéler une vérité sur soi, sur le monde et sur Dieu qui soit seconde par rapport aux stricts énoncés, renvoyant dès lors à un absolu (la vérité de Dieu) ou un non-lié (c'est l'étymologie du mot : absolu) qui ne fait pas nombre avec le temps, l'espace ou l'être du monde.

La vérité sur Dieu et sur l'humain n'est donc pas ici liée à des " contenus ", mais peut-être à des postures, des manières de se poser dans l'être, ou de se poser par rapport à soi et à l'altérité, des manières de se rapporter et d'être rapporté à soi et à l'altérité (le monde et Dieu, les dieux ou l'absence de dieu[x]).

Nous allons maintenant rassembler les principales données des Écritures bibliques et de l'histoire doctrinale chrétienne touchant notre thématique, en faisant état des représentations sanctionnées ou proposées, tout en supposant déjà, dans la mise en place, la dissociation qu'on vient d'évoquer entre " contenu " et posture engagée.

\subsection{Données bibliques}

La perspective mise en scène au plan biblique dit la consistance propre et l'autonomie du monde et du réel. La création apparaît irréductible à Dieu, tout en étant dite bonne et bénie : elle en vient, certes, mais selon une constitution qui est fonction d'une différence originaire et déterminante, non selon une génération par exemple, fût-ce par diversification (différente de Dieu, la création est d'emblée globale et structurée). Elle apparaît également irréductible à l'humain : elle le précède en tout point, et l'humain la reçoit venue d'ailleurs et déjà formée : il ne l'invente ni n'en poursuit le processus, mais en répond et s'inscrit dans sa différence, pour le meilleur ou pour le pire, pour la vie ou pour la mort ; en ce sens, on peut parler du monde comme "tiers » entre l'humain et Dieu (comme d'ailleurs entre l'humain et l'humain, soi ou autrui).

L'origine du monde est ici, stricto sensu, inconnue (elle peut être pensée, évoquée, mais non décrite), elle est autre et interdite : la créa- 
tion vient de Dieu (elle est ex nibilo dira-t-on ultérieurement, dans des registres différents), et elle est rapportée au tranché d'une Parole, non à une homogénéité d'être ou d'essence. Du coup — c'est en effet lié l'humain reçoit la création comme donné déjà cultivé, structuré par la Loi (mythologiquement : l'arbre du milieu du jardin) et traversé de la réalité du mal (le serpent), hors de toute linéarité de provenance et d'advenir.

Si la différence - non la continuité ou l'homogénéité — est ici originaire, ce n'est pas du fait d'une imperfection (ou, pire, d'un péché) ; la différence joue au contraire comme pivot, décisif, au cœur tant de ma réception du monde comme création (voir la mise en scène d'Adam en Gn 2-3) que de la dramatique croyante elle-même (voir, en christianisme, la dénonciation d'une hypostase de la loi, à laquelle on s'assimile, dans la mise en scène de Jésus face aux Pharisiens et la reprise par Paul du même motif à partir de la croix). Ainsi originaire, la différence est, potentiellement ou au titre d'une condition requise, fructueuse.

Pour une théologie s'efforçant de reprendre une telle mise en place des positions et des enjeux, renvoyer à Dieu ne devrait dès lors pas être au principe d'une totalisation, au regard du monde, sa réalité profane et extérieure, ni non plus au regard des réalités de la foi, aussi bien l'Église et ses données symboliques propres, son ordre d'appartenance et de représentations, que le fait du croire comme tel ${ }^{5}$.

C'est dire que, pour une telle théologie toujours, le monde donné est d'abord contingence : le monde est donné en dehors de toute immédiateté de l'humain et du principe dont il vit, son origine et sa vérité. Un monde comme contingence donnée - comprenant l'énigme d'un mal originaire - en appelle, en matière de vérité dernière ou de Dieu, non à une logique de continuité et de synthèse organisatrice, mais à une logique du surcroît ou de l'excès. À mon sens, se dessine alors, pour l'humain, la dramatique d'une réponse qui va se décider, théologiquement et spirituellement, "devant Dieu ", mais qui se joue, concrètement, au travers de la manière dont l'humain répond du monde donné. L'humain répond moins à Dieu, directement, qu'il ne répond de la manière dont il se pose dans le monde, de

5. Pour plus de développements sur ce point, cf. mon L'Excès $d u$ croire. Expérience du monde et accès à soi, Paris, Desclée de Brouwer, 1990. 
la manière dont il reçoit la création ou de la manière qu'il a de s'en croire l'origine et le maître, le connaisseur et le possesseur ( être comme des dieux " soufflait le serpent à Adam ou "transforme les pierres en pains ${ }^{6}$, dit Satan à Jésus typé comme nouvel Adam).

Notons que c'est au cœur d'un monde qui le précède et le dépasse que l'humain - homme et femme - est dit " image de Dieu ». Le lien à Dieu n'est en effet pas à chercher aux confins (avec les dérivations ou les marches d'approche que cela peut supposer ou entraîner), pas plus du côté d'un premier " commencement » (sur ce point, le monde est ex nibilo) que du côté d'un terme "dernier » (le monde passe et est destiné au néant).

Si le rapport à Dieu se noue au cœur du monde, à son propos et au gré d'une reprise assumée en responsabilité singulière - en régime incarné et personnel - , se marque alors, sur le pôle de l'humain également un excès (un manque aussi : l'homme a à être) qui renvoie à ce Dieu origine et fin, lui-même en excès (en absence aussi : Dieu a ici à être révélé). Cet excès auquel l'humain est assigné répond, en son ordre, à l'excès qu'est Dieu, tant au lieu de l'origine, en discontinuité avec les logiques de la nature (l'énigme du mal s'y loge), qu'au lieu de la fin, également en discontinuité avec les logiques de la nature et de l'histoire (les renversements de type apocalyptiques s'y insèrent). C'est pourquoi la thématique du croire est irréductible, avec ses promesses et ses perversions; mais elle se greffe directement sur ce qu'est l'existence même, au présent : elle en radicalise l'enjeu et les cristallisations concrètes effectives.

Précisons enfin que parce que le monde ne fait nombre ni avec ce qui peut être confessé comme vérité première et dernière (la vérité de Dieu), ni avec l'humain (toujours singulier), l'avènement d'existence qui s'y inscrit prend corps dans un espace ouvert et une irréductible pluralité. De même, le réel se donne là sur un horizon universel, tout aussi irréductible aux particularités de chacun (ce déploiement pluriel et cet horizon universel donnent consistance concrète à l'autonomie de la création).

Globalement, la Bible chrétienne raconte une longue histoire, focalisée sur les relations entre des hommes et leur Dieu, historiquement

6. On aura remarqué que la tentation — profonde ou spirituelle — n'a pas ici la forme d'une action éthiquement répréhensible... 
traversée de ruptures et de reprises, d'exil et de retour, de loi et d'appel prophétique, d'accomplissements, de transgressions et de relances, de bénédictions secrètes et d'imprévus. Or, cette histoire est encadrée de textes disant, en amont, une origine radicale, différente de l'élection et de ses suites, et, en aval, une fin elle aussi radicale et différente de l'histoire des croyants et de ce qui s'y joue. Prêtons-y attention : le patriarche ancêtre du peuple (juif) ou le "père des croyants " (chrétien), Abraham, n'est pas le premier homme, Adam. Et le Messie reconnu en Jésus n'ouvre pas directement ni sans rupture l'achèvement final (le "Royaume ») : c'est un Messie crucifié au cœur du monde et aux prises avec une histoire d'hommes. On dit ici un accomplissement, certes, mais il échappe à l'histoire visible (il est dit dans la foi) : le " Royaume " n'est pas là, et l'histoire n'y conduit pas naturellement, ni linéairement. Il est foncièrement d'un autre ordre. Comme symétriquement, en amont, la création du monde est d'un autre ordre que l'histoire qui va s'y déployer.

Bibliquement, l'histoire du monde et des hommes est encadrée par des récits d'origine et de fin qui ne lui sont pas linéairement accrochés, ni ne lui sont homogènes. Ils évoquent, en forme d'images (mythes et apocalypses), des données d'un autre ordre et qui sanctionnent une discontinuité : une origine vers laquelle on ne saurait remonter, ni soi ni l'histoire des hommes, et une fin ou un accomplissement qu'on ne saurait toucher ou réaliser, ni soi ni l'histoire des hommes.

Vus sous un certain angle, les deux textes de Genèse 1 et de Genèse 2-3 peuvent faire doublet. Leur articulation dans le texte biblique d'ensemble dit néanmoins encore autre chose que la texture propre à chacun. Elle fait que se présente d'abord un cadre cosmique (Gn 1), suspendu à Dieu et achevé en son ordre : les sept jours, sanctionnés par le sabbat où Dieu se " repose ", avec, au centre de cette fresque originaire et achevée en son ordre, une place marquée pour l'homme, une place centrale et cadrée justement. Décroché, hors de la continuité d'un déroulement descriptif ou narratif, vient ensuite le second texte (Gn 2-3), non plus la présentation d'un cadre cosmique originaire, mais le récit d'une genèse de l'humain, au cœur d'un jardin déjà planté, la genèse d'un humain sollicité par un mal, le serpent, dont on ne sait pas d'où il peut bien venir (il ne vient pas en tout cas tel quel de la fresque de Gn 1, de la création bonne et bénie de Dieu). 
Entre les deux récits, il y a, dans l'ordre biblique reçu, hiatus. C'est que se tiennent, au lieu de l'origine, deux types de données. L'une est de frappe cosmique, sans événement à proprement parler. C'est une donnée matricielle, celle des éléments du monde, ici différenciés. Et il y a une autre donnée, celle d'une effectuation de l'humain au cœur du monde, de frappe historique, même si c'est d'un temps immémorial ou paradigmatique. À côté d'une matrice faite des éléments du monde, un moment généalogique est ainsi inscrit dans l'originaire. L'humain - chacun d'entre nous - est précédé d'une donne cosmique, mais il est aussi précédé, sur un autre plan, d'une histoire d'engendrement qui laisse une trace. L'une et l'autre sont par-delà ma mémoire, mais c'est en fonction de l'une comme de l'autre, enfouies tout en étant bien là, que je peux naître à moi-même, dans le temps, en fragilité, exposé aux autres, mal assuré, tenté ou investi de divers vertiges, et accompagné ou surplombé d'une promesse à habiter.

\section{3 Éléments de la réception historique cbrétienne}

L'Antiquité tardive - véritable matrice de notre civilisation occidentale — a fait l'objet de nombreuses propositions de salut (gnoses diverses, dont les manichéismes; reprises des mystères ou de l'orphisme ; culte de Mithra ; etc.). Le christianisme fut l'une d'elles. Mais il prit sa forme propre et ses caractéristiques en cristallisant et en pensant une proposition de salut qui, loin de déserter, de nier ou de disqualifier l'ordre de la création (la réalité des choses, dans leur consistance propre et sous un horizon commun à tous, " universel »), s'y articule au contraire, intrinsèquement.

En lien avec la compréhension d'un salut articulé à la création, le christianisme s'est battu ici pour une conception spécifique de Dieu. Dieu y est compris comme extérieur au monde et non (à l'instar des dieux de certaines mythologies) comme le doublet de ses chatoiements enchanteurs et multiples, mais sans que cela conduise à une dévalorisation du réel et de l'bumain (pour laquelle serait seul vrai ce qui relève d'un spirituel au-delà de l'être). En d'autres termes, Dieu et le monde sont différents, mais ont tous deux valeur et importance, dans leur ordre propre à chaque fois. Le Dieu chrétien est origine première et dernière, mais il se tient au principe d'une création qui advient sous une figure à la fois finie et éminente, ou selon une structuration singulière de l'étendue spatiale et du flux temporel. 
À l'encontre de bien des gnoses, le christianisme ne renvoie pas la matière - ni le temps, ni le corps - à un principe mauvais, le salut étant seul rapporté à un principe bon. Mais il met en avant l'ordre d'un mystère, différencié : le mystère d'une intrigue inscrite au cour de la création, celle d'une liberté couplée à une séduction d'infini, malheureuse, et promise à une subversion possible, pour le meilleur.

Dieu a ici statut de gratuité. Il n'est ni lié au monde, ni nécessaire; il est fait d'une surabondance. Il est d'un autre ordre, d'un autre espace et d'un autre temps (ou autre que l'espace et que le temps, le temps et la création étant inhérents l'un à l'autre). Dieu relève d'une asymétrie, mais il peut - ou peut justement - se tenir au cœur de tous nos présents. Décroché du monde et de ses lois naturelles, il est au principe d'un surplus. Saint Augustin dira Dieu radicalement transcendant (" plus haut que ce qui est le plus haut ») tout en étant radicalement immanent ("plus intime que ce qui m'est le plus intime », Confessions III, VI, 11).

Entre Dieu et la création, entre Dieu et l'humain, il n'y a pas d'arrière-plan homogène, pas de coordonnées ni de mesures communes. Le christianisme ne suppose ni ne conduit à un monolithisme; de même qu'il coupe avec tout aménagement, plus ou moins équilibré, de forces dualistes. Il dit un Dieu autre, un Dieu qui n'est pas la forme vraie des choses, ni la plus achevée, ni leur somme, potentielle ou cachée. Classiquement, Dieu est amour (non confiscable) et grâce (imprévisible).

Lorsque au Moyen Âge, Thomas d'Aquin — un penseur typique et qui fera ultérieurement figure de référence emblématique, en régime catholique surtout — use du mot " être ", ce n'est pas au sens, neutre, de ce qui est simplement là et peut être disposé devant nous (ce qui est dans l'espace ou y prend place, comme le penseront les premiers philosophes de la modernité7, ou ce que les philosophes appellent techniquement les " étants »). Au contraire, par « être ", et à propos de la création, Thomas entend toujours, dès l'abord, une réalité qui a forme et qualité, qui présente une complexité et qui vit de relation.

Par «être » - l'être posé par Dieu ou rapporté à Dieu —, on entend, dans la synthèse proposée par Thomas d'Aquin au cœur du Moyen Âge, un donné, fini et éminent, qui, dit techniquement,

7. « Ce qui est étendu » (res extensa), dit Descartes dans la première partie du XVII ${ }^{\mathrm{e}}$ siècle. 
« existe » ou est « en acte ». N'a ici d'être au sens fort — devant Dieu, pour Dieu ou selon Dieu - que ce qui relève d'un ordre relationnel justement : ce qui reçoit l'exister et, à partir de là, se reprend sur soi. La création est relation, relation de grâce et non de nécessité, une relation inscrite, à titre constitutif, dans le fait d'exister.

En cela, la « cause » de l'être qu'est Dieu - Dieu comme origine de l'être — n'est pas le premier terme d'une série qui s'ensuit, mais une réalité transcendante, autre, au principe d'une relation, fragile (en « disproportion » dit la tradition), prenant corps au cœur du donné, pour le faire être : le faire exister ou être ce qui lui est donné d'être.

Une objectivation du monde fonctionnant selon des lois universelles et une compréhension de la cause première comme premier commencement (un Dieu premier horloger ou donnant une chiquenaude initiale) constituent l'arrière-fond sur lequel les théodicées vont voir le jour. Cet arrière-fond est aussi celui de ce qu'on appelle, couramment, la théologie naturelle. Or, son affirmation ou son refus me semblent dessiner la constellation des débats dans lesquels se meuvent la théologie et la foi chrétiennes depuis environ un siècle et demi.

La théologie naturelle a été sanctionnée au Concile de Vatican I dans sa Constitution Dei Filius (1870). Parmi ses citations les plus nettes, notons : "Dieu, principe et fin de toutes choses, peut être connu avec certitude par la lumière naturelle de la raison humaine à partir des choses créées" (chap. 2). Se reprend ici, pour le Concile, ce qui relève de la théologie de la création. La révélation n'y a pas de pertinence. Le Concile entend répondre au rationalisme, mais sur son terrain, sans déplacement des termes. La déclaration citée se situe sur le plan de l'objectivité telle que l'entend spontanément l'homme moderne, même s'il s'agit d'une objectivité où, bien sûr, l'Église ne voit pas les choses de la même manière que les scientifiques. Il y a conflit ${ }^{8}$, mais chacun est au même niveau.

En effet si ce qui relève de la thématique de la création se joue dorénavant dans les termes qu'on vient de souligner, la révélation relèvera de données supplémentaires, celles - c'est sans surprise - du

8. Canon 2, 1: «Si quelqu'un dit que le Dieu unique et véritable, notre Créateur et Seigneur, ne peut être connu avec certitude par ses œuvres grâce à la lumière naturelle de la raison humaine, qu'il soit anathème ». 
salut et de l'Église : "Outre les vérités que la raison naturelle peut atteindre, nous sont proposés à croire les mystères cachés en Dieu, qui ne peuvent être connus s'ils ne sont divinement révélés " (chap. 4). On se trouve ici à l'opposé de toute objectivité et de toute raison. Concernant ces vérités qui ne sont plus celles de Dieu comme Créateur, mais de ce qui s'ajoute et réclame révélation, spécifique et hors raison, le Concile doit mettre les points sur les i : " Nous croyons vraies les choses qu'il nous a révélées, non pas à cause de leur vérité intrinsèque perçue par la lumière naturelle de la raison, mais à cause de l'autorité de Dieu ». Le fondement est celui d'une pure intervention extérieure. En toute logique est dès lors requise, " la soumission plénière de notre intelligence et de notre volonté » (chap.3).

À la même époque, des théologiens protestants libéraux élaboraient aussi ce qui peut passer pour une théologie naturelle (ou qui peut jouer une fonction analogue), mais selon d'autres coordonnées, à partir de la psychologie par exemple ou d'une anthropologie religieuse. Les registres sont alors ceux de l'homme et de son déploiement historique et social. A l'opposé, des courants franchement conservateurs, néo-calvinistes par exemple, pourraient fournir des parallèles protestants très nets à ce qui se cristallise à Vatican I.

Après la cassure culturelle qu'a représentée la Première Guerre mondiale, le $\mathrm{XX}^{\mathrm{e}}$ siècle a pris, majoritairement, le contre-pied de la théologie naturelle qu'on vient d'évoquer. D'abord et surtout en terrain protestant, mais ensuite suivi, avec des nuances, par bien des théologiens catholiques.

Avec des hommes comme Karl Barth ou Rudolf Bultmann - mais ils expriment une tendance ecclésiale et culturelle dominante - , c'est délibérément un motif de la révélation transcrit en termes d'interpellation et de "kérygme » qui vient au centre, reprenant non la thématique de la création, mais celle du salut : du péché (compris comme auto-enfermement) et de la grâce (entendue comme irruption d'altérité et de transcendance).

La théologie de la création passe alors à l'arrière-plan. Elle se trouve soit abandonnée, soit comprise comme le prolongement, par extension, de l'expérience d'une libération, expérience seule fondatrice, mais intra-historique justement et non située aux commencements. De l'expérience d'un Dieu libérateur lors de la sortie d'Égypte, on serait passé à la confession que ce Dieu est maître de tout l'univers, 
comme du mystère pascal on aurait été conduit à une confession plaçant le Christ au cœur du mystère de la création globale. C'est ainsi que les exégètes lisent alors la Bible (Gerhard von Rad pour l'Ancien Testament par exemple). Quant à Barth, il comprend la création comme «alliance» (Dogmatique, début de la partie III, $\mathbb{S} 41$ ).

La force de cette réaction ne doit pas être négligée. C'est le refus d'entendre la création comme cadre extérieur, quasi neutralisé, et la volonté de retrouver une problématique franchement théologique et croyante qui, comme telle, se laisse toujours à nouveau instruire par les mystères du salut (le mystère pascal, la christologie, les sacrements, l'expérience de foi). La construction de Barth est ici, encore une fois, explicite : elle met délibérément en correspondance salut et création, au titre d'une «alliance interne » et d'une "alliance externe » qui renvoient l'une à l'autre.

Qu'il y ait là une opposition nette à l'égard du propos de Vatican I mis en exergue, tous en sont conscients. L'expression " théologie naturelle » est d'ailleurs devenue une valeur péjorative et discriminante chez la majorité des théologiens et pasteurs de plusieurs générations. Quant à Barth lui-même, il a expressément déclaré que la théologie naturelle n'était " pas une des nombreuses hérésies possibles, mais l'hérésie par excellence ${ }^{9}$, une déclaration qu'on peut rapprocher d'une autre de ses affirmations célèbres : si l'" on ne peut pas devenir catholique ", ce n'est pas à cause du pape, de Marie ou de "toutes les autres raisons qu'on peut avoir », mais à cause de "l'analogie de l'être » (la ressemblance entre le créé et le créateur qui permettrait de remonter du premier au second) qui est une "invention de l'Antéchrist ${ }^{10}$.

L'opposition est nette. J'entends néanmoins la dépasser. J'ai critiqué la position cristallisée à Vatican I et signalé le bien-fondé de la réaction qui en prend le contre-pied. Il reste à dire en quoi cette réaction ne me paraît pas satisfaisante.

Soulignons d'abord que la faiblesse vient justement de ce qu'il s'agit là, en bonne partie, d'une réaction, avec ce que cela peut entraîner de polarisation et d'unilatéralité. S'abandonnant au désir - légitime d'articuler création et salut (ou création et christologie), on a perdu de

9. Dogmatique II/1* , Genève, Labor et Fides, 1956 (1940), p. 140.

10. Ibid., I/1*, 1953 (1932), p. XII. 
vue ce qui pouvait constituer les traits spécifiques et la nécessité de la thématique de la création. On a oublié en quoi elle n'est pas qu'un doublet ou qu'une simple extension du motif du salut (de la libération ou de la christologie), mais un aspect irréductible, pouvant apporter des correctifs à une confession du salut prise pour elle-même, détachée de la thématique de la création. De même réciproquement, le motif du salut peut apporter des correctifs à ce que proposerait une thématique de la création indûment autonomisée ou rationalisée.

La tâche est claire. C'est celle de notre génération. Elle correspond en outre à une demande culturelle et sociale (et, à mes yeux, à une nécessité ecclésiale). Il s'agit de rien de moins que de dépasser l'opposition signalée et de retravailler chacun des deux termes qui se font face : mettre donc en perspective et problématiser ce qui est central dans la vision chrétienne de la création d'une part, ne pas s'installer dans une focalisation sur le seul moment du salut de l'autre. Et c'est, du coup, renouer avec la confrontation, difficile mais nécessaire et fructueuse, de la foi et de la raison (une confrontation que la théologie dominante $\mathrm{du} \mathrm{XX}^{\mathrm{e}}$ siècle a souvent abandonnée) ; par-delà — les points sont liés ou sont les différentes faces d'une même donnée d'ensemble - c'est renouer avec une théologie qui sache mieux penser et faire valoir sa pertinence dans la société globale et face à ses interrogations. On l'esquissera dans le point 5.

\section{Une proposition de balisage théologique}

Entre les affirmations de la science sur les commencements et la consistance ou les structurations de l'univers, de la vie et de l'humain d'une part, et celles de la Bible — comme celles d'autres traditions religieuses d'ailleurs - , d'autre part, il y a un net hiatus. On aurait tort de le sous-estimer. Ce hiatus atteint les représentations, les descriptions ou les narrations ${ }^{11}$ qu'on

11. On notera que la forme narrative n'est pas propre aux seuls discours religieux, cf. Hubert ReEves, Patience dans l'azur. L'évolution cosmique, Paris, Seuil, 1981 ; ID., Dernières nouvelles du cosmos, Paris, Seuil : t. I : Vers la première seconde, 1994 et t. II : La première seconde, 1995 ; Stephen HAWKING, Une brève histoire du temps. Du big-bang aux trous noirs (1988), Paris Flammarion, 1989 ; Jean HeIDMANN, L'odyssée cosmique. Quel destin pour l'Univers? Paris, Denoël, 1986 ; plus ancien, Carl Friedrich von WeIZAECKer, Die Geschichte der Natur, Zürich, Hirzel, 1948. 
peut donner des commencements et des structurations. Ce n'est pas rien. Cela pourrait certes être relativisé et dépassé : les représentations cosmologiques ne sont ni homogènes ni constantes, dans la Bible comme dans la tradition théologique chrétienne. Mais l'écart entre le discours scientifique et discours de foi est en réalité plus irréductible : il affecte la place de l'humain dans le monde (centrale et spécifique dans la tradition biblique et théologique, tardive, dérivée et sans autonomie pour la science) et, du coup, son rapport possible avec un Dieu créateur du monde (déterminant pour la même tradition biblique et théologique, sans assignation dicible ni pensable pour la science).

Dans les milieux théologiques et ecclésiaux - hors dogmatisation catholique indûe ou fondamentalisme protestant - , on parle souvent de complémentarité entre discours scientifiques et discours de foi ${ }^{12}$. A mon sens, un tel projet n'est pas à récuser comme tel. Mon questionnement porte seulement sur le type de complémentarités : la définition et la circonscription de chacun des discours en jeu d'une part, à l'interne donc, la manière de les articuler ou de faire jouer leurs frontières d'autre part, à l'externe. Pour le dire d'un trait, il me paraît souvent que les recherches de complémentarités ne poussent pas assez radicalement la logique de chacun des discours en jeu et que - c'est lié - la vision d'ensemble proposée au final est trop harmonisante, sa pertinence et son statut exacts restant au demeurant un peu flous ${ }^{13}$.

12. Comme exemple récent, citons Jean-Michel MALDAMÉ, Le Christ pour l'univers. Pour une collaboration entre science et foi, Paris, Desclée, 1998.

13. Notons au passage que si la théologie ou les religions ne sont, ni à même, ni habilitées à se prononcer sur les structures de la matière et de la vie, leurs fonctionnements et leurs évolutions, toute détermination à ce niveau et tout finalisme apparaissent répudiés, comme toute vision, à ce niveau toujours, d'une " humanisation du cosmos », y compris le " principe anthropique » (cf. John D. BARRow et Frank J. Tipler, The Antropic Cosmological Principle, Oxford, Oxford Univ. Press, 1988 et ID., L'homme et le cosmos. Le principe anthropique en astrophysique moderne [entretiens avec Marie-Odile MonchicourT], postface de Hubert Reeves, Paris, Imago, 1984), cette probable "réintroduction de l'anthropocentrisme dans la science ", dit Marc LACHIEZE-REY, op. cit., p. 554 et, plus largement, p. 549-557). En outre, s'il est vrai que la science ne se prononce pas sur une origine absolue ou en dehors de l'univers existant et peut reconnaître ce qui est inaccessible, j'aimerais 
Au vu de ce que j'ai rappelé au point 2 , j'aimerais tout particulièrement valider, quant à une mise en place d'ensemble, la marque d'inconnu, de secret ou d'énigme qui est attachée tant aux "commencements " qu'aux "fins » du monde et de la vie. C'est en fonction de cet inconnu - dont la face positive ne peut que se penser comme contingence donnée et reprise sur soi - que se décide l'avènement de l'humain en vérité. Corrélativement, cet inconnu foncier ne peut qu'aller de pair avec un monde irréductiblement pluriel, socialement, culturellement et religieusement; si le christianisme entend se proposer comme monothéisme, ce ne saurait dès lors être celui d'un absolu comme fondement et sanction premiers et derniers de tout ce qui est, mais celui d'un absolu qui, supposant et réclamant l'autonomie et la pluralité du monde ${ }^{14}$, est lié à l'avènement d'une existence singulière, tout à la fois exposée et traversée tant par une promesse possible subvertissant la vie et son donné cosmique que par des perversions propres.

Précisons que dans la perspective d'ensemble proposée, ce n'est pas seulement une dogmatisation catholique indue et le fondamentalisme protestant qui doivent être récusés comme " mise en série " ${ }^{15}$ indue de ce qu'on peut dire et savoir sur l'état du monde, son évolution, et de ce que la théologie peut penser sous la catégorie d' "origine » (renvoyant, classiquement, $\mathrm{au}$ " caractère incommunicable de la puissance créatrice de Dieu»). Doivent aussi à mon sens être critiquées, les visions qui en appellent à une relation de quasi-partenariat entre Dieu et l'homme, le Créateur et la créature ${ }^{16}$, en vue d'une poursuite ou d'un

souligner, contre une certaine apologétique, que l'inaccessible n'est pas ici tangentiel, mais principiel : il n'y a pas inachèvement, ouverture ou imperfection, mais discontinuité ou rupture d'ordres.

14. Sur ce point, cf. Le christianisme est-il un monothéisme? (G. EMERY et P. GISEL éd.), Genève, Labor et Fides, sous presse.

15. Je suis ici, en profonde consonance, avec Christoph THEObALD, « La théologie de la création en question ", Recherches de science religieuse, (1993), p. 613-641.

16. On hérite là d'une conjoncture typiquement moderne des problèmes : la validation quasi transcendantale, chez Schleiermacher et contre Fichte, d'une "précédence » du monde et de l'exister sur le sujet, purement conçu comme acte, cristallise un moment classique de riposte, cf. Le christianisme est-il un monothéisme? (G. EMERY et P. GISEL éd.), début de la partie III. 
prolongement - voire d'un achèvement — de la création ${ }^{17}$. Certains textes de Vatican II n'en sont pas indemnes.

C'est en démarcation de ces théologies modernes ou contemporaines que j'ai pour ma part toujours souligné l'achèvement de la création, en son ordre, et la dissymétrie entre ce qui se dit là de "précédence » ou de surplomb irréductible à l'homme, à son temps et à son espace, d'une part, et, de l'autre, ce qui s'énonce en termes d'histoire, d'autonomie ou de liberté. C'est à mon sens là que se jouent la spécificité et la raison d'être du thème de la création en théologie chrétienne, irréductible à la thématique du salut, venant s'y articuler dans la différence ou la contrebalancer, alors que, laissée à elle-même, une problématique du salut ou ses reprises sécularisées risque de sombrer dans ses propres perversions anthropocentriques (une relecture de Nietzsche sera ici de grand profit).

Soulignons enfin que la pluralité indiquée au plan du monde et de l'histoire humaine ira de pair avec une diversité des instances et des rationalités qui président aux ordres, différents, que sont le politique, le civil, le droit ou le moral, comme, justement, le savoir scientifique, l'expression culturelle et le religieux. Cette diversification des instances et des espaces évoqués les laisse irréductibles les uns aux autres, donc autonomes dans leur ordre propre à chaque fois, et même non " coordonnables" - encore moins intégrables - selon une logique unique ou selon une homogénéité sociale quelconque, même idéale.

\section{Foi et savoir : quelles articulations et pour quels enjeux ?}

Discours religieux d'une part, discours scientifique de l'autre. J'ai esquissé certains des termes d'une mise en perspective, qui organise leur prise en compte concomitante. En contrepoint et avant d'aller plus loin, il convient peut-être de passer brièvement en revue différents modèles d'articulations proposés et certains des enjeux qui peuvent y être liés.

17. Chez les théologiens, à titre d'exemple : Alexandre GANOCZY, Homme créateur-Dieu créateur (1976), Paris, Cerf, 1979. On récusera également les variantes qui, chez Jürgen Moltmann par exemple, proposent, en lien avec une " périchorèse " trinitaire foncière, une quasi-intégration de l'humain dans la vie de Dieu et une « inhabitation » de Dieu dans le monde. 
Auparavant pourtant, il me paraît nécessaire de faire un peu d'histoire. C'est que les rapports entre croire et savoir n'ont pas toujours été les mêmes. Ils forment à chaque fois une conjoncture donnée, située.

\subsection{Savoir et croire : brève mise en perspective historique}

Ni le savoir ni le croire n'ont toujours été pensés et investis de la même manière. Leurs limites respectives, comme leur teneur propre - leur type de rationalité, de déploiement, de validité ou de plausibilité, ce qu'on en attendait et ce qui pouvait en être reconnu — ont varié. En outre, savoir et croire font couple. À telle vision - tel statut, telle organisation et telle limite - du savoir correspond telle vision - possible ou impossible - du croire.

L'Antiquité chrétienne et le Moyen Âge ont vécu d'une vision du monde complexe et différenciée, mais synthétique, où le savoir en appelait à un croire (dans la distinction) et où le croire s'articulait à un savoir (reconnu dans son indépendance, ne serait-ce qu'au vu de ses sources non-chrétiennes). Globalement, on a vécu ici d'une vision (une "métaphysique ") où "substance " et "qualité » sont indissociables (les choses ont telle ou telle qualité qui les définit), l'ensemble étant inséré dans une structure cosmique de la "participation " qui commande la spiritualité d'une origine, d'une fin et d'une raison des choses, récapitulative et transcendante.

Or, sauf revanches ésotériques, cette synthèse se casse à l'aube des Temps modernes, sur le plan de la culture reconnue et socialement opérante. La connaissance se fait à la fois plus expérimentale (active et pratique) et mathématique (déductive et combinatoire). Le sujet humain dès lors mis en scène est un sujet libre et créateur, quasi desserti de l'ensemble organique d'un cosmos intégré, à recevoir, habiter et contempler. Les formes du savoir sont critiques et dissociatives ; elles donnent lieu à exaltation et à angoisse.

Aux premiers temps de la modernité occidentale (de la Renaissance aux Lumières), la science est analytique : elle décompose (cf. Descartes). La vision globale est celle d'un mécanisme. La physique n'est plus celle, antérieure, des " qualités » (intrinsèques et greffées sur les éléments fondamentaux du cosmos, leurs jeux de sympathie et d'antipathie) et où chaque chose avait son "lieu " (auparavant, la pierre ne retombe pas sur la terre à cause de propriétés mesurables et de la loi de la gravitation, mais parce que tel est son lieu d'origine, où 
elle ne peut que revenir et reposer). Le jugement, enfin, est la conclusion d'une procédure correctement - méthodiquement - menée, et il va à l'encontre de l'imagination (cf. Spinoza), que l'on renvoie alors à la superstition, celle qui marque le fond des traditions et des institutions, dont il faut se libérer.

La nature est ici éprouvée, culturellement et existentiellement, comme lieu de l'absence de Dieu. Non plus lourde de significations et de symboles, mais froide, objectivée et soumise à mesure quantitative. S'il y a un créateur, il est renvoyé au commencement (il a créé le mécanisme de départ).

En profondeur, et quoi qu'il en soit de certaines modifications au plan strictement scientifique, nous héritons socio-culturellement de tout cela (c'est le premier moment de notre modernité, et il demeure à l'arrière-plan), tout particulièrement en matière de représentation quant au savoir, donc de place et de forme imparties au croire.

La modernité a vécu d'une idéologie du progrès et de l'idée d'une substitution, inéluctable et profitable, de la science (seule vraie) à la religion, aux mythes et aux fables, réputés simplement faux (ainsi Auguste Comte, fondateur d'une "Église positiviste » et auteur, en 1852, d'un Catéchisme positiviste, fait se succéder, selon une voie de rationalisation progressive justement, l'âge théologique, l'âge métaphysique et l'âge scientifique, dernier et définitif).

Aujourd'hui, le positivisme est battu en brèche. Au moins dans sa forme la plus monolithique et la plus idéologique. C'est que les promesses de transparence et de libération sociale qui pouvaient lui être liées, à l'encontre des obscurantismes aliénants, se sont révélées ambivalentes quand ce n'était pas franchement trompeuses. L'épopée moderne ne trouve plus en elle-même sa légitimation. Elle doit faire face à des résistances imprévues (bonnes ou mauvaises), à des complexités et des pluralités peut-être irréductibles, à des revanches ou des retours. La modernité doute. Et l'on se prend à parler, çà et là, de postmodernité, où savoir et croire sont revisités. 


\subsection{De quelques modèles d'articulation entre science et foi}

\subsubsection{Conflit entre la science et la foi}

D'un côté, on pensera en termes de substitution. De l'erreur (les traditions religieuses et la foi) à la vérité (les connaissances scientifiques et les procédures du savoir). De l'autre, en strict vis-à-vis, on estimera devoir défendre les affirmations religieuses sur le plan même des représentations et des contenus proposés.

Le Syllabus romain de 1864 ( «Recueil renfermant les principales erreurs de notre temps »), des aspects centraux de Vatican I et la réaction anti-moderniste (à partir de 1907) chez les catholiques, le néocalvinisme qui apparaît aux environs de 1880 ou le fondamentalisme chez les protestants, fournissent de bonnes illustrations de cette attitude. Dans leurs apparitions comme dans leur logique interne, ils sont tous, n'en déplaise aux intentions de leurs acteurs, des produits modernes, nés sur sol moderne et de forme moderne ${ }^{18}$.

\subsubsection{D'une complémentarité entre la science et la foi à un refus d'articulation}

En matière de complémentarité, la répartition la plus fréquente est d'impartir à la science le comment (la description des phénomènes de la nature et de leurs lois internes) et à la foi ou à la religion le pourquoi (le sens des mêmes phénomènes). Science et foi sont ici complémentaires parce qu'elles ne sont pas du même ordre ou ne répondent pas aux mêmes questions. Chacune, la science comme la foi, a alors une vue partielle et la complémentarité est possible, mais il ne s'agit pas là d'une articulation sur fond homogène.

De cette complémentarité sur fond de distinction, on passe facilement à une séparation plus radicale. On a refusé de mélanger les compétences ou craint les interférences; on va dès lors adopter une attitude qui veut que les scientifiques soient toujours plus scientifiques et les croyants ou les théologiens toujours plus occupés de la foi et de sa spécificité (Dieu et son interpellation radicale, sans analogie avec

18. Ce n'est probablement pas par hasard que l'on trouve en général plus de fondamentalistes parmi les personnes de formation scientifique que parmi les personnes de formation humaniste classique (littérature, histoire, philosophie), phénomène également repérable en islam, par exemple. 
l'ordre du monde). Du coup, la distinction qui supposait complémentarité se fait séparation et fonctionne, de fait, comme immunisation.

\subsubsection{Modèles de convergence}

Des modèles de convergence, il y en a de plusieurs formes. Certains défendent aujourd'hui un principe dit anthropique (cf. ci-dessus n. 13). Pour l'essentiel, il consiste à valoriser le fait ou l'impression que tout l'univers semble être disposé en vue de l'homme et que, sur ce point, foi chrétienne et données scientifiques peuvent se rejoindre, en ayant emprunté des chemins différents bien sûr. Il y aurait donc, inscrite au cœur de l'univers, une intentionnalité secrète.

D'autres - deuxième exemple - présentent un type de convergence en mettant en avant des analogies entre vision scientifique et vision religieuse. Ainsi certains auteurs soulignent-ils une affinité entre les concepts de la physique quantique et le tao (c'est le cas du physicien Fritjof Capra) ou, plus largement, entre science contemporaine et religions orientales. On pourrait aussi citer, la " gnose de Princeton " (Raymond Ruyer), d'il y a environ un quart de siècle.

Dans un contexte et une intention analogues, on assiste encore, aujourd'hui, à une exploitation des données sur fond d'échec d'un paradigme matérialiste unidimensionnel, selon lequel le savoir objectif de la modernité classique viendrait entièrement à bout des réalités du monde et de l'humain ; d'où une mise en avant de phénomènes spirituels, parapsychologiques ou paramédicaux.

Enfin, on peut faire référence à la théologie du Process, fort répandue aux États-Unis, qui, à partir des travaux du philosophe et mathématicien Alfred North Whitehead (1861-1947), repense la création en rompant avec tout modèle que surplomberait la notion d'une éternité immobile, pour inscrire Dieu lui-même dans le devenir (un "procès ») où il est partie prenante de l'avènement, toujours temporel ou " actuel ", des choses. Là encore, on vise une convergence entre la science et la foi qui puisse présenter une plausibilité culturelle adaptée à notre temps.

Indépendamment des questions liées au fait même de vouloir établir des convergences (la légitimité et la possibilité d'un tel programme), j'avoue ne pas bien saisir les différences de fond qu'il y aurait à faire voir les analogies mentionnées ici entre représentations 
religieuses et tel aspect de la science contemporaine par rapport aux tentatives plus anciennes, élaborées à l'enseigne des théodicées modernes ou des constructions de théologie naturelle. Là aussi, on entendait jouer sur des analogies entre la religion (chrétienne en l'occurrence) et les savoirs de l'époque. Les visions proposées aujourd'hui sont simplement adaptées aux données de notre temps. On est ainsi passé de l'articulation d'un Dieu créateur, cause extérieure, foncièrement simple en lui-même (une certaine forme de monothéisme était alors privilégiée), avec une nature objectivée selon les paradigmes du rationalisme de la modernité classique à l'articulation d'un Dieu plus complexe, dont l'action est présente, continue et diversifiée, avec un cosmos lui aussi plus complexe, traversé d'interactions et d'interdépendances diverses. Le passage d'une vision à l'autre est culturellement significatif, mais modifie-t-il la logique de fond ? ou a-t-on simplement changé de concordisme, parce qu'il fallait bien s'adapter?

\subsection{Récapitulation}

La science d'aujourd'hui souligne l'absence de tout finalisme repérable sur le plan du déploiement de l'univers et de la vie, de même qu'elle casse tout anthropocentrisme. Globalement, elle ignore tout ordre de nécessité préétabli, ne connaissant que des événements survenus sans raison, mais désormais irréversibles et qui constituent un ensemble donné, avec ses régulations internes et son ouverture foncière.

Se lancer dans un conflit science/foi - sur le plan des représentations du monde, de la vie et de l'humain - est une bataille perdue d'avance, à moins de tomber dans une position sectaire ou autocentrée. Mais, surtout, c'est se méprendre tant sur ce que sont les données religieuses que sur ce qu'est la foi, avec ce qui s'y engage du sujet humain. En outre, c'est ne pas pouvoir répondre de la diversité des discours religieux.

Passons sur les tentatives de concordisme, voulant établir des similitudes de même niveau entre ce que peuvent mettre respectivement en avant la science et la foi religieuse. La critique contre elles me paraît aisée : focalisation sur des données ou des informations coupées de leur contexte d'énonciation et de fonctionnement ; sélections scientifiquement arbitraires et, au demeurant, précaires dans le temps, alors même qu'on en espérait le contraire ; non-validation, voire refoule- 
ment, de ce que peut représenter le fait de la religion et de la foi comme tel, avec ses problèmes, ses enjeux, ses lois et ses risques propres.

J'ai opté pour une distinction entre la posture humaine que suppose l'engagement de foi - son type de rapport à soi, au monde et à Dieu - et les représentations qu'elle propose à chaque fois. Cette option me paraît commandée par ce qu'est la tradition chrétienne dans son ensemble, texte biblique compris. La théologie chrétienne ne part pas - en principe ! - d'une conception ou d'une vision déployée du monde, de l'homme et de Dieu, pour tenter de les valider face à d'autres conceptions ou visions ; elle travaille au cœur du présent social, culturel et religieux qui est le sien à chaque moment de l'histoire. Et elle est là aux prises avec les questions de tous, pour y inscrire un type d'interrogation, de problématisation et d'ouverture, qui soit spécifique certes et instruit par l'histoire qui la précède, mais qui embraie, dès le départ, sur les données du monde et de l'humain comme tels, des données problématiques, ouvertes, en débat, voire en conflit. Or, les questions de l'origine, de l'être et de l'humain sont précisément des questions qui relèvent de données communes, universelles en ce sens, inscrites au cœur de l'humain et toujours récurrentes.

La spécification de chacune des perspectives, notamment religieuse et scientifique, est une autolimitation quant à la portée de l'angle de vue de chacune (aucune d'entre elles n'épuise à elle seule l'ensemble du donné). L'existence d'autres perspectives sur le même objet pour autant qu'elle soit reconnue, empêche un auto-enfermement et une idéologisation interne. Il est ainsi salutaire, pour la foi et la théologie, que la science vienne remettre en cause les représentations qui avaient été les leurs : cela ne peut que les relancer dans l'interrogation touchant le statut de leurs représentations (le meilleur des énoncés croyants peut toujours devenir idolâtrique) comme dans l'approfondissement de ce qui les constitue dans leur vérité propre (le croire) en lien avec leur objet propre (Dieu ou l'absolu).

Avancer que l'homme doit problématiser le lien noué avec des représentations du monde et se défaire de faux savoirs ne signifie pas qu'il puisse vivre sans images. Ce serait faire preuve d'idéalisme. L'homme se dit dans des mythes et des images, au demeurant non indifférents ni interchangeables, et dès lors instructifs ; toute constitution d'identité, personnelle ou collective, se noue au travers d'images, de récits ou de mythes. Il faut seulement reconnaître que ces 
images et visions sont changeantes, inscrites en une culture et une mémoire à chaque fois, et les faire travailler à ce niveau.

Pour moi, le recours à Dieu n'a pas à fonctionner comme une explication première et dernière de l'ensemble des choses, de la naissance du cosmos et de ses lois, ou de l'apparition de l'homme et de son fonctionnement. Le rapport entre Dieu et le monde n'est pas direct. D'où notamment une place pour des discours différents, scientifiques et religieux, et, à l'intérieur de la science, pour une histoire et des controverses, ainsi que, au sein du religieux, pour des pluralités ${ }^{19}$. Entre Dieu et le monde, il y a discontinuité, décalage, asymétrie. Dieu n'est pas homogène à notre espace et à notre temps. C'est d'ailleurs pourquoi la foi peut tenir qu'il est présent - et absent - à tous les temps de l'histoire.

Dieu n'est pas non plus à évoquer comme la raison interne du cosmos et de la vie ou leur logique secrète. Le rapport entre Dieu et la création n'est pas fait d'échanges secrets, d'émanation ou de redoublement, d'homologie, d'embrassement ou de fusion. Le monde n'est pas de Dieu ni en Dieu : il a sa consistance et son autonomie propres.

À cela correspond une vision du monde; réciproquement d'ailleurs. Ce sera celle d'un monde reçu dans sa contingence (le fait d'être là, sans qu'on puisse en dire la raison), un monde dont l'homme ne peut faire le tour, un monde qui ne lui est pas entièrement approprié, même si l'homme se plaît à l'habiter, un monde qui, pour l'homme, est inséré dans de la démesure, avec son poids d'énigme, de malheur aussi, et de malheur inexplicable — qu'il n'y a pas à expliquer, encore moins à justifier.

Quant à l'humain, dans la conscience qu'il a de lui-même l'image de lui qu'il peut assumer ou l'identité qu'il peut recevoir et construire - , il faudra en souligner la différence à l'égard de Dieu comme à l'égard du monde et du strict biologique, sauf à ce que cet humain se perde, n'accède pas à soi, à son être. Inscrit en contingence et partie prenante du monde, l'humain se recevra comme sujet qui répondra de lui-même en répondant de ce monde hors de la raison qui

19. Notons bien que pluralité religieuse et modifications ou controverses scientifiques ne sont pas à mettre en parallèle : elles n'ont pas le même statut, ni ne se pensent et ne s'arbitrent de la même manière. 
le précède et le dépasse. Une tâche ouverte par l'absence de nécessité soulignée et délimitée par cette absence. L'humain y verra un défi et une vocation, une difficulté certes (il n'y a ni programme, ni garantie), mais un surcroît possible aussi : une sorte d'intensification d'être, dont il n'a pas la maîtrise, mais qui passe par lui et par ce qu'il lui est donné d'exister. Ici, l'humain est d'une forme et d'une teneur qui, tout à la fois réclament le donné du monde (son immanence) et le dépassent selon une autre logique (transcendante).

L'image qu'on peut ainsi se faire du monde et de l'humain impliquera une défense de l'humain dans sa finitude, ses possibilités et ses tentations. On saura que la nature ou la vie n'est pas sacrée, ni, en principe, intouchable; mais on en usera pour le meilleur, et ici, le meilleur, c'est celui d'une mesure humaine. On saura qu'il est bon pour l'homme qu'il reconnaisse qu'il n'est pas maitre de tout et qu'il dépend d'équilibres qu'il ne peut impunément détruire et qu'il est bon qu'il naisse et se déploie dans la différence, biologiquement, culturellement, spirituellement.

L'irréductibilité du cosmos à l'humain me paraît devoir être assumée, tout comme l'irréductible différence entre savoir du monde et foi religieuse, ainsi que, du coup, l'irréductible pluralité du religieux. Ces différences sont même requises : elles radicalisent et donnent tout son poids à l'avènement de l'humain en vérité. Contre toute intégration totalisante, que ce soit du côté du monde, du cosmos ou de la vie (ou encore de l'histoire), ou que ce soit du côté du divin.

En tout cela, on tiendra que la vie n'est pas l'humain, ni l'humain la vie. La vie est, pour l'homme, une condition d'existence, donnée et fragile, à préserver (non à sacraliser). Quant à l'humain, il en appelle à d'autres paramètres. Au cœur de la vie, l'humain marque une singularité où s'articule sa valeur propre. Seul cet humain peut être figure ou parabole d'absolu, et dans la différence des personnes à chaque fois. Si la vie est condition à préserver, l'humain est à organiser dans sa différence propre et dans les infinies particularités qui s'y font jour. En profondeur, la force et la valeur de l'humain, assumant sa particularité à chaque fois, seront données en proportion de son ouverture à ce qu'il n'est pas : le monde ou la nature, le biologique, d'autres manières d'être homme, l'absolu bien sûr ; hors de cela, l'organisation de l'humain se fait sourdement totalitaire. 


\section{Quel statut pour la théologie ?}

La foi et la théologie sont liées à la tradition d'une manière de se poser dans l'être. C'est que le religieux, fait de matrices symboliques et de données socio-culturelles larges, se cristallise dans des religions historiques (elles vont de pair avec des formes de civilisations). Cette tradition est constamment traversée d'acculturation et de recomposition, et l'on peut donc dire qu'elle est en condition d'odyssée. Liées à une tradition, la foi et la théologie ont ainsi part à un phénomène socioculturel d'institutionnalisation, avec ses frontières et ses marges (l'apocryphe, l'ésotérique, etc.), ses divers types aussi (l'Église, la secte et la mystique, pour reprendre Weber et Troeltsch), ainsi qu'à des partitions, elles aussi socioculturelles et changeantes, où se spécifient le croire, le savoir, la morale ou l'esthétique et où s'organise un ordre social global.

La foi et la théologie ne peuvent dès lors se limiter à une défense ou une réinterprétation d'énoncés confessés (un dieu, une forme de médiation, de ritualité, etc.) ou d'événements allégués (via des histoires symboliquement organisées). La théologie doit, greffée sur le mouvement de tradition qu'on vient d'évoquer - une tradition comprise comme la prise en charge de données globalement humaines et prenant toujours à nouveau corps dans un socioculturel particulier -, se faire critique et réflexive. Tout en s'inscrivant dans la culture et les quêtes spirituelles $d u$ temps (ce qu'elle a fait au cœur de l'Antiquité tardive, moment classique de sa constitution à proprement parler, à distance et en différence du moment qu'elle se reconnaît ou se construit comme référence, le moment biblique), la théologie ne cessera de développer en même temps une réflexion attachée au statut de la vérité dont elle entend rendre compte et raison (elle y a pourvu au cœur de la même Antiquité tardive via un moment qu'on dira ensuite de théologie négative, greffé sur ses affirmations et ses positivités, permettant de les approfondir, "spirituellement ", et d'éviter qu'elles ne soient occasion d'idolâtrie). Cette vérité s'inscrit en outre délibérément dans le cadre d'une problématique communément partagée (pour en rester au moment classique de l'Antiquité tardive, celle de la question du divin, de son rapport à l'être ou de son statut d'au-delà de l'être, celle des systèmes de médiations et d'intermédiaires, etc.) et non, comme cela a commencé à l'être avec le début des Temps modernes, en catholicisme comme en protestantisme, l'attachement à une 
" révélation ", légitimant et accréditant une institution spécifique, de type ecclésial.

Globalement, je tiens dès lors que la théologie comme discipline a pour lieu et objet, d'un côté, la symbolisation du monde, de l'existence humaine et de l'espace social, et, de l'autre, le rapport à $l^{\prime} a b s o l u^{20}$. Tel me paraît être en effet la double face de l'ordre humain des croyances. La théologie y est assignée et s'y déploie comme une discipline qui réfléchit, selon un axe propre, aux productions idéologiques liées aux institutionnalisations humaines, leurs symboliques organisationnelles (assurant des jeux de normativité) et leurs mises en place de références ou d'idéaux en matière de légitimation (passant par constructions de l'imaginaire). J'ajoute qu'en modernité, une telle perspective conduit à passer par une histoire des diverses relations qui se sont nouées entre l'Église comme institutionnalisation du religieux ("hérésies" ou autres contrepoints inclus) et la société comme espace socioculturel, présidant à des circonscriptions déterminées à chaque fois, des instances de légitimité et des types de rationalité reconnus. Cette histoire se fera dès lors généalogie de la modernité et $d u$ destin $d u$ religieux en modernité (sécularisation, ersatz idéologiques, nouveaux mouvements religieux, recompositions, etc.), s'efforçant d'y proposer des orientations claires quant à leur limites de validité et quant à leur forces et à leurs faiblesses possibles (les secondes sont toujours le revers des premières) et de pertinence globale (non réservée à un groupe spécifique, fût-il ouvert, ni aux adhérents d'un message à transmettre, reçu de l'extérieur, dût-il concerner tout le monde). Pour qu'elles soient de pertinence globale, il aura fallu qu'elles soient, dès le départ et constitutivement, la prise en charge spécifiée bien sûr — d'une question de tous. Par rapport à une certaine manière de comprendre la foi et la théologie, c'est peut-être là aussi une odyssée ; elle me paraît faire cohérence avec ce que j’ai esquissé à l'occasion des rapports entre savoir et foi à propos des commencements (de l'origine ?) et des structurations (de l'être ?) du monde.

20. Pour plus de développement, je me permets de renvoyer à mon ouvrage, La théologie face aux sciences religieuses. Différences et interactions, Genève, Labor et Fides, 1999. 


\section{RÉSUMÉ}

La présente contribution cherche à saisir l'impact des changements de représentations du cosmos sur la théologie. Après avoir rappelé certaines données scientifiques et présenter un aperçu des données bibliques et chrétiennes en la matière, l'auteur fait une proposition de balisage théologique au regard de la non-homogénéité ou des contradictions qui se dégagent de la confrontation de la science et de la Bible. Il fait alors un retour sur les types d'articulation possibles entre le savoir scientifique et le fait de croire. Il présente enfin un aperçu touchant la question du statut de la théologie.

\section{ABSTRACT}

The present contribution seeks to grasp the impact of the change in our representation of the cosmos. The author first recalls some of the scientific data and presents an overview of the christian and biblical data on the subject. He also proposes reference points to guide the way of theology while taking into account the lack of homogeneity and the contradictions that appear when scientific and biblical data are confronted. He then looks at different possible ways of articulating scientific knowledge and faith. He finally gives an outline concerning the status of theology 